\title{
A search for neutrinos from Gamma Ray Bursts with the IceCube Neutrino Detector
}

\section{The IceCube Collaboration ${ }^{1}$}

${ }^{1}$ http://icecube.wisc.edu/collaboration/authors/icrc15_icecube

E-mail: martin.casierevub.ac.be

\begin{abstract}
The origin of the Ultra-High Energy Cosmic Rays (UHECRs) is still unknown. Gamma-Ray Bursts (GRBs) are generally presented as possible candidates to host progenitors producing such UHECRs. However, the exact physical processes underlying GRBs are yet not fully understood. If GRBs are (partly) responsible for the observed UHECRs, they have to contain a hadronic component, and consequently high-energy neutrinos must also be produced. In this case, large scale neutrino observatories on Earth, such as the cubic kilometer IceCube Neutrino Observatory located at the South Pole, should be able to detect them. We present a new search method based on two separate datasets: the long $\left(T_{90}>2 \mathrm{~s}\right)$ and short $\left(T_{90} \leq 2 \mathrm{~s}\right)$ GRBs. They will be treated separately in order to obtain the best possible sensitivity as the commonly accepted picture is that long GRBs and short GRBs have a different progenitor. Our studies will be based on different event selections and specific statistical methods.
\end{abstract}

Corresponding authors: L. Brayeur*, M. Casier*, G. Golup and N. van Eijndhoven Vrije Universiteit Brussel, Dienst ELEM

Pleinlaan 2, B-1050 Brussels, Belgium

The 34th International Cosmic Ray Conference,

30 July- 6 August, 2015

The Hague, The Netherlands

${ }^{*}$ Speaker. 


\section{Introduction}

Cosmic rays are observed in a very broad range of energies, up to about $10^{20} \mathrm{eV}$ and the origin of the highest energy part is still puzzling. Gamma-ray bursts (GRBs) have been proposed $[1,2]$ as promising candidates to host progenitors producing the Ultra-High Energy Cosmic Rays (UHECRs) because of their extremely large energy release (of the order of $\sim 10^{54} \times \frac{\Omega_{\gamma}}{4 \pi} \sim 10^{51} \mathrm{ergs}$, where $\Omega_{\gamma}$ is the solid angle into which the gamma-rays are beamed) over time scales of only $\sim$ $10^{-3}-10^{3} \mathrm{~s}$. In this context, a mechanism for particle acceleration during these cataclysmic events has been developed $[3,4,5]$. It is known as the fireball model, in which gamma-rays are produced by the dissipation of kinetic energy in an ultra-relativistic fireball. If GRBs accelerate protons through sufficiently efficient processes, they could account for most or all of the UHECR flux [2]. In this case, a neutrino signal is also expected because the accelerated protons and ambient photons will interact through the $\Delta$-resonance and produce charged pions : $p+\gamma \rightarrow \Delta^{+} \rightarrow n+\pi^{+}$. These charged pions will then decay leptonically via $\pi^{+} \rightarrow \mu^{+}+v_{\mu}$ followed by $\mu^{+} \rightarrow e^{+}+v_{e}+\bar{v}_{\mu}$. Therefore, the fireball should result in a flux of high-energy $v_{\mu}$ and $v_{e}$, coincident with the gamma rays [6]. Such a flux should be detectable on Earth by a sufficiently large detector. Although only $v_{\mu}$ and $v_{e}$ are produced at the source, on Earth the total neutrino flux is expected to be equally composed of three neutrino flavors because of neutrino oscillations. Neutrinos correlated with GRBs would therefore be a "smoking-gun" signal for UHECR acceleration in GRBs but up to now, no prompt neutrino signal has been detected either by IceCube or by Antares [7, 8, 9, 10].

We describe in the following a new search method for detecting these neutrinos using the IceCube Neutrino Observatory. IceCube is a cubic-kilometer neutrino detector installed in the ice at the geographic South Pole [11] between depths of $1450 \mathrm{~m}$ and $2450 \mathrm{~m}$. Detector construction started in 2005 and finished in 2010. Neutrino reconstruction relies on the optical detection of Cherenkov radiation emitted by secondary particles produced in neutrino interactions in the surrounding ice or the nearby bedrock. We aim to analyse four years of data of the fully completed detector (from May 13th, 2011 to May 18th, 2015) for all the GRBs located in the Northern hemisphere during this period.

\section{Gamma ray bursts}

GRBs are short, unexpected and principally non-thermal bursts of $\gamma$-rays. In terms of duration, a "typical" GRB lasts for $\mathscr{O}(10 \mathrm{~s})$ but it can vary from the millisecond scale to thousands of seconds, as can be seen in Fig. 1. Early hints [12] provided indications that GRBs can be separated into 2 categories according to their duration, which was confirmed with the observations performed with the BATSE satellite [13]. The 2 distinctive types are: short GRBs (SGRBs) with $T_{90} \leq 2 \mathrm{~s}$ and long GRBs (LGRBs) with $T_{90}>2 \mathrm{~s}$, where $T_{90}$ is defined as the time needed to accumulate from $5 \%$ to $95 \%$ of the fluence in the $50-300 \mathrm{keV}$ band. This separation is clearly visible in Fig. 1. This segregation of GRBs cannot be attributed to an instrumental artefact but it represents a real physical property [13]. Both subpopulations are indeed isotropically distributed on the sky as expected from two independent uniform distributions of extra-galactic objects. The observed GRB population consists of about $30 \%$ of SGRBs and $70 \%$ of LGRBs. 


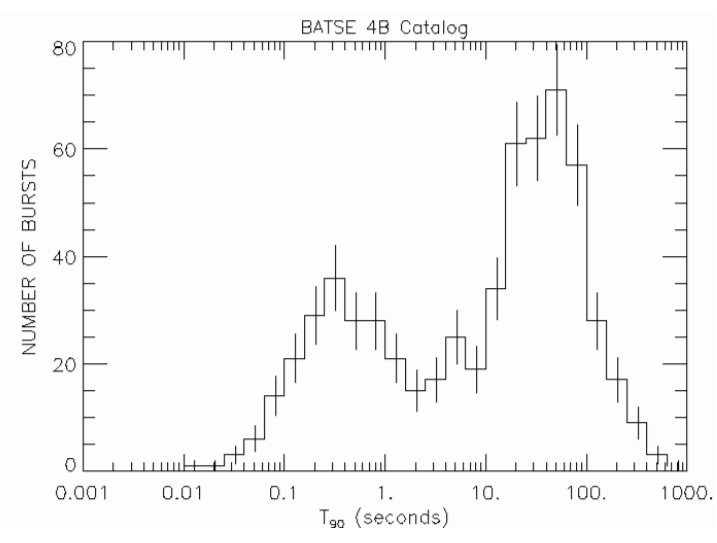

Figure 1: Distribution of $T_{90}$ for 1234 GRBs in the BATSE 4B Catalog. From [14].

Evidence points to GRBs being the visible part of catastrophic energy release in stellar massive objects. However, a complete understanding of the different stages involved in the GRB phenomenon is still missing. In this context, the observation of two duration classes may indicate different progenitors. It is believed that the LGRBs are almost surely associated to the "death" of a massive star, i.e. the collapse of its core, leading to the so-called collapsar model [15]. This is supported by associations of GRBs with supernova events [16]. The picture is less settled yet for the SGRBs, but there are some observational indications $[17,18]$ that SGRBs could be associated with compact binary mergers [19] of two neutron stars or of a neutron star with a black hole. However, the generic evolution of the explosion after the initial energy injection is most probably independent of the progenitor. The "inner engine" would only have some influence on the variability of the light curves of the GRB [20].

As explained in the introduction, neutrinos are also expected to be created by GRBs. These neutrinos may be produced during three consecutive phases with respect to the gamma flash: precursor (before the actual GRB), prompt (during the emission of gamma-rays) and afterglow. This variety of signals makes the timing signature of the neutrino very complex and therefore makes it difficult to link with a gamma observation. We also expect a broad range of possible emission spectra for each of these specific phases [14]. For SGRBs, it has been shown that the produced neutrino spectrum is expected to be harder than $E^{-2}$ because the very high accelerating gradients needed in these objects, will accelerate the muons before their decay [21]. We therefore intend to perform an analysis based on very generic models. In this context, the physical information of a separation of the GRBs in two subclasses in terms of their duration offers the opportunity to enhance the significance for a time spectral analysis.

\section{Data samples and event selection}

For developing the event selection of our analysis according to the IceCube blindness policy, we use an off-source burn sample as background estimation composed of 48 data samples of 2 consecutive hours during 4 years (from May 13th, 2011 to May 18th, 2015) of real data without a known GRB. Each data sample corresponds to each month of the considered period, to account for the seasonal variations in the cosmic ray intensity [22]. The signal is modelled by a Monte Carlo 
simulation of a generic expected signal spectrum of $E^{-2}$, corresponding to the well-known Fermi acceleration mechanism. The analysis has been optimised for "well-reconstructed" signal ${ }^{1}$.

The first phase of the event selection consists of a newly developed criterion which selects the muon tracks that are most likely up-going in the detector, i.e. particles coming from the Northern hemisphere. This method is based on the combination of several track reconstruction methods in order to select more efficiently the up-going tracks. After this first selection, a Boosted Decision Tree (BDT) is trained on the data and allows us to reduce the background data to the $\mathrm{mHz}$ scale (atmospheric neutrino flux scale) while keeping up to $\sim 90 \%$ of the well-reconstructed simulated signal events. This leads to an effective area shown in Fig. 2, where this observable is defined as the ratio of the observed event rate and the incoming neutrino flux. As such the effective area, obtained from simulations, provides a means to relate our observations with the actual neutrino flux. We see that we improve the event selection compared with the latest IceCube analysis [10] with a gain especially in the low energy regime. This will lead to an increase of events that pass the developed event selection. Furthermore, the $1 \sigma$ angular resolution of the passing signal events is $\Delta \Psi=1.35^{\circ}$, which is comparable with the previous analysis [10]. Note that the effective area and angular resolution values are given after a cut on the BDT score leading to a signal efficiency of $85 \%$ and a signal purity of $86 \%$.

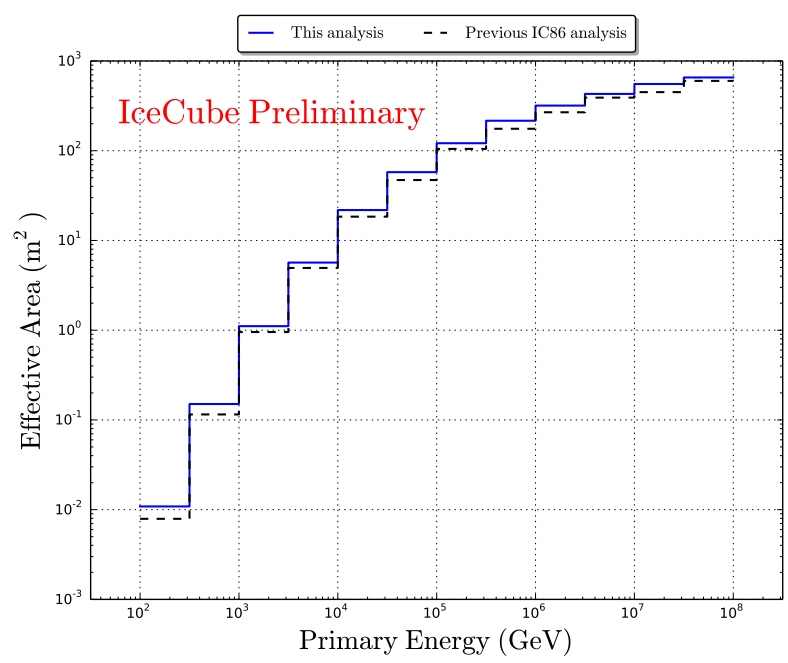

Figure 2: Effective area of the expected neutrino signal for an $E^{-2}$ spectrum as a function of the neutrino energy. The blue line represents this analysis and the black dotted line the latest IceCube result performed with the full detector [10].

\section{Statistical analysis}

\subsection{Short GRBs}

In the case of short GRBs, a simple cut and count experiment has been performed. This

\footnotetext{
${ }^{1}$ In IceCube, each physical event is reconstructed by different algorithms, leading to several possible reconstructed tracks. Here, we define well-reconstructed signal as having one of these reconstructed tracks in a $5^{\circ}$ angular window around the simulated direction.
} 
method aims to detect possible prompt neutrinos from GRBs. This analysis is based on a stacking procedure for all the SGRBs in the considered period, which amounts to 82 for the 4 considered years.

The analysis is composed of two different cuts that are performed on the data:

- Time cut: a time window of $4 \mathrm{~s}$ beginning $1 \mathrm{~s}$ before the starting time of the GRB ;

- Spatial cut: an angular window given by the square root of the quadratic sum of the GRB angular uncertainty and the reconstruction precision.

The passing events are then stacked for all GRBs and the test statistic applied is the Poisson distribution.

In order to determine the mean value of the background Poisson distribution and to extract the method sensitivity and the discovery potential limits for each of the BDT score cut thresholds, a null hypothesis experiment is performed. It consists of pseudo-experiments that have been performed by randomising the GRB azimuth angles and the event starting times. Finally, signal is injected to determine the BDT cut by optimising for discovery the fluence normalisation $F_{0}$, for a generic $E^{-2}$ spectrum and for a Waxman-Bahcall spectrum with a first energy break at $\varepsilon=10^{6} \mathrm{GeV}$ [10]. This procedure is applied for different BDT score thresholds, ranging from 0.05 to 0.25 in steps of 0.01 . The 4 years results are presented in Fig. 3. The optimisation of the BDT cut for obtaining the best sensitivity instead of optimisation for discovery gives very similar results.
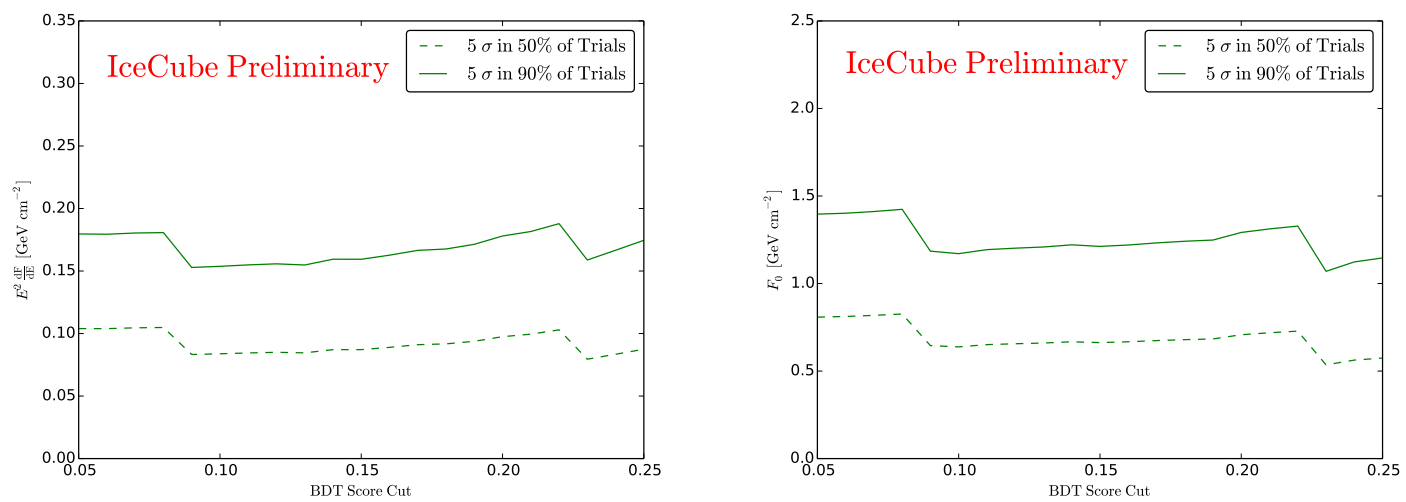

Figure 3: Fluence normalisation factor in function of the BDT score threshold for generic $E^{-2}$ spectrum (left) and in the case of a Waxman-Bahcall spectrum [10] (right).

The results shown in Fig. 3 are competitive compared to previous IceCube analyses [10] and they are the first discovery potential limits reported for a short GRB search. We see that whereas the chosen spectrum has an impact on the fluence normalisation factor it is independent for the optimisation of the BDT cut. We propose a cut at $\mathrm{BDT}_{\mathrm{score}} \sim 0.10$. This is the loosest cut which is still in the discovery potential signal optimum plateau for both spectrums.

An extension of the method, analysing the time difference between observed events, will be performed in the future to be also sensitive to precursor and afterglow neutrinos. 


\subsection{Long GRBs}

In order to determine the best statistical method for the detection of neutrinos from LGRBs, several methods were compared using a toy model simulation of the arrival times and locations of background and signal events. Previous searches using the complete IceCube detector have been tailored for prompt neutrinos [10]. This analysis aims at being sensitive to the three neutrino emission phases (precursor, prompt and afterglow) mentioned before. For this purpose, a time window around the GRB trigger time as well as an angular window around the GRB position are defined. One is limited in the choice of such windows since a too large window would increase the background and reduce the sensitivity of the method. Hence, an analysis cannot be sensitive to all possible precursor and afterglow times.

In this analysis, we have chosen to consider a time window of one hour around the trigger time of GRBs and an angular window of $15^{\circ}$ around each GRB position. These choices are the results of an optimisation based on the different statistical methods used. Our toy model simulation generates signal and background events corresponding to the 491 LGRBs of the four years data sample taking into account position and reconstruction uncertainties, duration and redshift effects. After the numerical event generation, the time and angular windows around the 491 simulated GRBs are stacked in a similar way as outlined in [24].

Two observables were simulated, the time between an observed muon and the associated GRB trigger time and the angular distance between the associated GRB position and the reconstructed direction of the events. The background event generation is uniform in time and isotropically distributed. For every signal event, the characteristics ( $\mathrm{T}_{90}$, angular uncertainty and redshift) of a GRB are chosen randomly from the list of GRBs.

The prompt signal event time is generated following a flat distribution during the $\gamma$ emission of the selected GRB $\left(\mathrm{T}_{90}\right)$ to which Gaussian tails are added on each side. In the precursor case, the signal event time is generated exactly 2 minutes before the GRB trigger time at the source. The actual event time of these precursor events varies as function of the GRB redshift. The angular distribution of signal events follows a Gaussian distribution with a standard deviation in which the median angular resolution of this analysis and the uncertainty on the selected GRB position are taken into account.

The method previously used in IceCube ${ }^{2}[10]$ and hereafter named "Likelihood" defines the test-statistic $T$ as

$$
T=\sup _{n_{s}}\left[-n_{s}+\sum_{i=1}^{N_{\text {event }}} \log \left(\frac{n_{s} S_{i}}{\left\langle n_{b}\right\rangle B_{i}}+1\right)\right],
$$

where $S=S_{\text {time }} \times S_{\text {angle }}$ indicates the signal Probability Density Function (PDF) and $B=B_{\text {time }} \times$ $B_{\text {angle }}$ the background one. $S_{\text {time }}$ and $S_{\text {angle }}$ are the same distributions as the ones used to generate the signal events. The background distribution $B_{\text {time }}$ is uniform in time and the background angular distribution $B_{\text {angle }}$ follows the solid angle effect. The parameters $n_{s}$ and $\left\langle n_{b}\right\rangle$ are the number of source neutrinos and the expected number of background events, respectively.

\footnotetext{
${ }^{2}$ In previous GRB analyses an energy spectrum was also considered in the Likelihood method to further distinguish background and signal events. We made the choice of not using an energy distribution in order to avoid biases towards higher energy events.
} 
The Likelihood method is compared to another method called hereafter "PLT", which is defined in [23]. This method considers the signal as a perturbation of the background. If the strength of this perturbation is $\eta$ and its location in time or in angle is $\theta$, we can define

$$
p(x \mid \eta, \theta)=(1-\eta) B(x)+\eta S(x \mid \theta)
$$

and

$$
S(\theta)=\left.\frac{\partial \log \left[\prod_{i=1}^{N_{\text {events }}} p\left(t_{i} \mid \eta, \theta\right)\right]}{\partial \eta}\right|_{\eta=0},
$$

where, as above, $B$ is the background PDF and $S$ is the signal one.

The test statistic is then defined as $T=\sup _{\theta} S(\theta)$. Such a definition can be interpreted as a "scanning" of the parameter $\theta$ in order to find the location where the perturbation is the strongest. This method should be sensitive to the three GRB phases while the Likelihood method can only be used for a prompt neutrino search.

The obtained results, shown in Fig. 4 indicate that the PLT method is only slightly more sensitive to a precursor signal than the Likelihood method. Furthermore, the PLT method can detect more efficiently weak signals but becomes less sensitive than the Likelihood method when the signal strength increases.
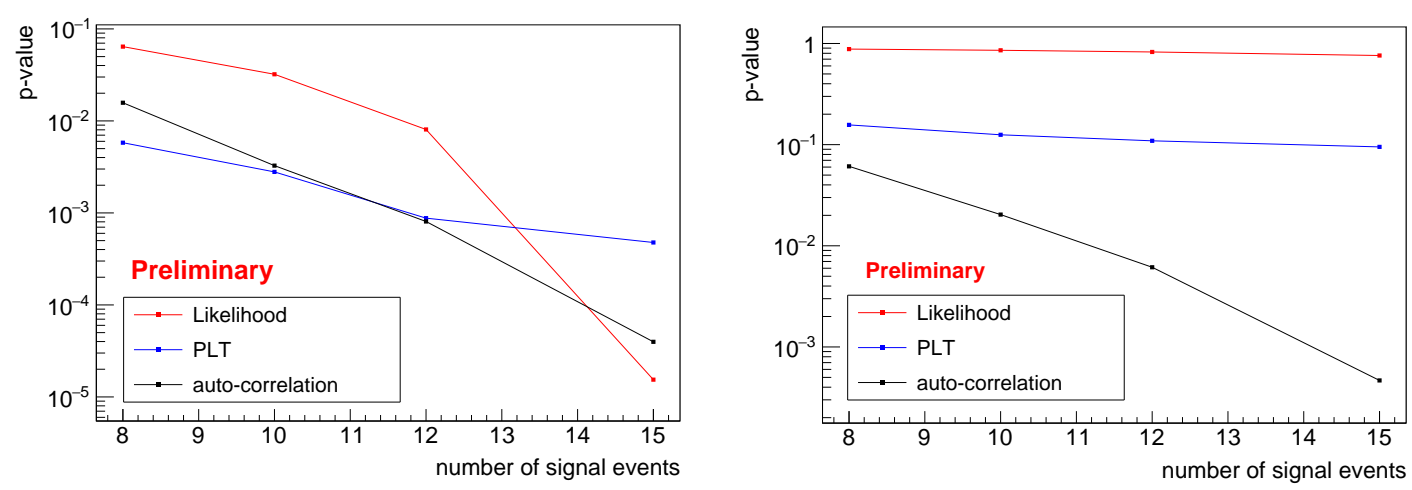

Figure 4: The p-value obtained from the three statistical methods described in the text, Likelihood in red, PLT in blue and the auto-correlation in black. The p-value is drawn in function of the number of signal events injected in the simulation. The left figure shows the prompt signal case and the right one considers a precursor signal. The background rate in this simulation is $3 \mathrm{mHz}$.

Another possibility under study consists of using an hybrid method using one of the likelihood methods described above for the angular part (for PLT, $\theta$ would then be the angle between the GRBs and the events) and the auto-correlation method for the time part. In the auto-correlation method we generate a distribution with the time differences between all possible event pairs. The distribution is then binned and the number of pairs per bin is compared to a background only simulation in order to extract the probability of having a certain number of pairs in a specific bin. The lowest probability of all the bins is reported as the test statistic. Since the sensitivity of the method depends on the binning choice, an optimisation has been performed in order to select the most appropriate binning. The auto-correlation is presented with the PLT and the Likelihood method on Fig. 4. 
The hybrid method would have the strength of a likelihood method for the angle distribution as well as a sensitivity for all GRB phases. The sensitivity of the auto-correlation method to both prompt and precursor events, as can be seen on Fig. 4 is due to the unnecessity of defining a signal PDF.

\section{Conclusion}

We have developed a new search for neutrinos from GRBs based on two dedicated methods for LGRBs and SGRBs. For the first time, the SGRBs has been studied separately and the results are promising. The discovery potential limits are competitive compared to previous IceCube analysis. An effort will be devoted in the near future to develop a statistical analysis sensitive to all the possible phases of neutrino emissions (precursor, prompt and afterglow).

In the case of the LGRBs search, in which signal events are expected in a broader time window, dedicated statistical tools are being investigated. As in the case of SGRBs, we aim to define a method sensitive to the three possible phases of neutrino emissions. We expect that the optimisation of the statistical method both for long and short GRBs combined with a more efficient event selection can result in a neutrino detection in relation with a GRB or in stringent upper-limits on the neutrino flux.

\section{References}

[1] M. Vietri, Astrophys. J. 453 (1995) 883.

[2] E. Waxman, Phys. Rev. Lett. 75 (1995) 386.

[3] A. Shemi and T. Piran, Astrophys. J. 453 (1990) L55.

[4] T. Piran, Rev. Mod. Phys. 76 (2004) 1143

[5] P. Mészáros, Rept. Prog. Phys. 69 (2006) 2259.

[6] E. Waxman and J. Bahcall, Phys. Rev. Lett. 78 (1997) 2292.

[7] IceCube Collaboration, R.Abbasi et al., Phys. Rev. Lett. 106 (2011) 141101.

[8] IceCube Collaboration, R.Abbasi et al., Nature 484 (2012) 351.

[9] Antares Collaboration, S. Adrián-Martínez et al., A\&A 559 (2013) A9.

[10] IceCube Collaboration, M. G. Aartsen et al., Astrophys. J. 805 (2015) L5.

[11] IceCube Collaboration, A. Achterberg et al., Astropart. Phys. 26 (2006) 155.

[12] K. Hurley, AIP Conf. Proc. 265 (1991) 3.

[13] C. Kouveliotou et al., Astrophys. J. 413 (1993) L101.

[14] J. K. Becker, Phys. Rept. 458 (2008) 173.

[15] S. E. Woosley, Astrophys. J. 405 (1993) 273.

[16] J. Hjorth et. al, Nature $\mathbf{4 2 3}$ (2003) 847-850.

[17] D. B. Fox et al., Nature 437 (2005) 845.

[18] E. Berger et al., Nature 438 (2005) 988.

[19] B. Paczynski, Astrophys. J. 308 (1986) L43.

[20] S. Kobayashi et al., Astrophys. J. 490 (1997) 92.

[21] S. R. Klein, Astrophys. J. 779 (2013) 106.

[22] IceCube Collaboration, S. Tilav et al., Atmospheric Variations as observed by IceCube, in Proc. of the 31st ICRC (2009) Lodz, Poland.

[23] R. Pilla, C. Loader, and C. Taylor, Phys. Rev. Lett. 95.

[24] N. van Eijndhoven, Astroparticle Physics 28 (2008) 540. 